In Press at Advances in Methods and Practices in Psychological Science

\title{
Examining Psychological Science through Systematic Meta-Method Analysis: A Call for Research
}

\author{
Malte Elson \\ Department of Psychology \\ Ruhr University Bochum
}

\begin{abstract}
Research synthesis is based on the assumption that when the same association between constructs is observed repeatedly in a field, the relationship is probably real, even if its exact magnitude can be debated. Yet this probability is not only a function of recurring results, but also of the quality and consistency in the empirical procedures that produced those results and that any meta-analysis necessarily inherits. Standardized protocols in data collection, analysis, and interpretation are important empirical properties, and a healthy sign of a discipline's maturity.

This manuscript proposes that meta-analyses as typically applied in psychology benefit from complementing their aggregates of observed effect sizes by systematically examining the standardization of methodology that deterministically produced them. Potential units of analyses are described and two examples are offered to illustrate the benefits of such efforts. Ideally, this synergetic approach emphasizes the role of methods in advancing theory by improving the quality of meta-analytic inferences.
\end{abstract}

Keywords: meta-analysis, meta-science, methodology, standardization, synthesis

Malte Elson, Psychology of Human Technology Interaction, Department of Psychology, Ruhr University Bochum, Universitätsstr. 150, 44801 Bochum. Tel: +49 23432 24032; E-Mail: malte.elson@rub.de This research is supported by the Digital Society research program funded by the Ministry of Culture and Science of North Rhine-Westphalia, Germany.

Acknowledgments. I sincerely thank Brenton Wiernik, reviewer of this manuscript at AMPPS, for one of the most thoughtful reviews I have ever seen. His comments and suggestions have substantially improved the paper. Further, I thank Nick Brown, Julia Eberle, James Ivory, Richard Morey, Julia Rohrer, and Tim van der Zee for comments on earlier versions of this paper. 
For the past eight years, psychologists have undergone a phase of intense selfexamination regarding standards and practices in the production, dissemination, and evaluation of knowledge. This critical scrutiny has covered integral aspects of psychological science's entire enterprise, including the maintenance of psychological theories (Ferguson \& Heene, 2012); the procedures by which given hypotheses are (dis-)confirmed or new research questions are generated and explored (Wagenmakers, Wetzels, Borsboom, van der Maas, \& Kievit, 2012); prevailing norms in the collection (Lakens \& Evers, 2014), analysis (Wagenmakers, Wetzels, Borsboom, \& van der Maas, 2011), reporting (Nuijten, Hartgerink, van Assen, Epskamp, \& Wicherts, 2016), and sharing (Wicherts, Bakker, \& Molenaar, 2011) of data; the consistency and accuracy of interpretations of observed effect sizes in various fields, e.g. industrial-organizational psychology and management (Wiernik, Kostal, Wilmot, Dilchert, \& Ones, 2017), personality and individual differences (Gignac \& Szodorai, 2016), language learning (Plonsky \& Oswald, 2014), and cognitive neuroscience (Szucs \& Ioannidis, 2017); publication practices (Nosek \& Bar-Anan, 2012), peer review, and quality management (Wicherts, Kievit, Bakker, \& Borsboom, 2012); reproducibility and replicability of research findings (Open Science Collaboration, 2015); the communication of these findings to the public (Sumner et al., 2014); and the contingent incentive structures under which academics operate (Nosek, Spies, \& Motyl, 2012).

An increasing number of failures to replicate empirical findings (Hagger et al., 2016; Open Science Collaboration, 2015; Wagenmakers et al., 2016) and scientists' admission to prevalent use of questionable research practices (John, Loewenstein, \& Prelec, 2012) that improve their odds of producing published research under the discipline's rigid preference for statistically significant results (Kühberger, Fritz, \& Scherndl, 2014) have worked in concert to erode trust in psychological science. Introductory textbooks, meta-analyses, literature reviews, and policy statements are all built on decades of (purportedly) cumulative research - but how reliable, how rigorous is this knowledge?

\section{Known Knowns and Known Unknowns}

Doubts about the reliability of psychological research have emerged with regards, in particular, to opaque data collection rules, flexibility in methods and measurement, and arbitrary analytical and computational procedures. This class of behaviors, which collectively facilitate the inflation of the probability of obtaining and subsequently publishing statistically significant results while omitting analyses yielding (potentially conflicting) nonsignificant results, is colloquially known as p-hacking (Simonsohn, Nelson, \& Simmons, 2014a). We know that these strategies can be quite effective, alone or in conjunction (see, e.g., Schönbrodt, 2016), and we know that psychologists use these strategies frequently (John et al., 2012; LeBel et al., 2013).

Since through $p$-hacking, by definition, relevant information is being omitted from the public record, identifying ex-post publication whether questionable research practices have shaped the results in published research reports (and if so, which types) is an intricate process. Outcome switching, optional stopping of data collection, and the use of multiple computation techniques for the same target variable are all known types of methodological flexibility, yet which of these behaviors and procedures were appropriated to produce inflated 
proportions of statistically significant results in any particular case is usually unknown. This problem is exacerbated when individual studies are combined in an attempt to synthesize a body of research (LeBel, McCarthy, Earp, Elson, \& Vanpaemel, 2018).

\section{Methodological Flexibility and Meta-Analyses}

Effective meta-analyses in psychology inform researchers about (1) the mean and variance of underlying population effects, (2) variability in effects across studies, and (3) potential moderator variables (Field \& Gillett, 2010). They do so by identifying a relevant population of research reports and selecting those meeting predefined inclusion criteria, such as methodological gold standards.

As $p$-hacking and the file drawer problem typically work in conjunction with each other $^{1}$, any attempt at (quantitative) research synthesis becomes quite cumbersome. Certainly, there is an abundance of bias estimation and correction techniques (Schmidt \& Hunter, 2015). Procedures such as Test of Insufficient Variance (Schimmack, 2014), puniform (van Assen, van Aert, \& Wicherts, 2014), p-curve (Simonsohn et al., 2014a, 2014b), funnel plot symmetry/trim-and-fill-based methods (Duval \& Tweedie, 2000), PET-PEESE (Stanley \& Doucouliagos, 2014), and various selection models (McShane, Böckenholt, \& Hansen, 2016) may reveal that some unknown bias is present in a population of statistical results. Yet when a body of empirical research is meta-analyzed, researchers might have trouble determining to what extent an obtained effect size estimate is affected by publication bias, methodological flexibility, or an interaction of the two. Furthermore, while such statistical estimation techniques are helpful in demonstrating that a body of research exhibits signs of bias or questionable research practices, they might be less effective in identifying their specific topography. Two recent simulation studies (Carter, Schönbrodt, Gervais, \& Hilgard, in press; Renkewitz \& Keiner, 2018) compared bias estimation techniques (in several models of publication bias and $p$-hacking), and report that (1) no method consistently outperforms the others, (2) performance of bias correction is poor under specific assumptions (that may be applicable to psychological research domains), and (3) researchers usually do not have the information required for selecting the (relatively) best technique.

Generally, research synthesis (meta-analytic or otherwise) is based on the assumption that when the same association between constructs is observed repeatedly in a field, the relationship is probably real, even if its exact magnitude can be debated (e.g., Ellis, 2010). Yet this probability is not only a function of recurring patterns of results on the construct level, but also of quality and consistency in the collection of empirical data, together with their measurement, transformation, computation, and reporting that may produce seemingly consistent results. Any meta-analysis necessarily inherits the properties of those empirical practices. With increasing diversity, the certainty whether studies in which the same constructs are purportedly assessed actually yield evidence on the same construct relationship decreases. Without a gold standard of assessment agreed upon by a scientific community, it can be quite difficult to estimate the consequences of inconsistent

\footnotetext{
${ }^{1}$ But see Nelson, Simmons, and Simonsohn (2018) who argue that the "file-drawer explanation" is incorrect, and that most failed studies are actually $p$-hacked into publication.
} 
methodological approaches. And the more the consistency of research outcomes in a given field depends on flexibility in methods and measures, the more the probability of a real association may approach zero.

\section{Meta-Method Analysis}

Meta-analyses might thus benefit from complementing their aggregates of observed effect sizes by systematically examining the standardization of methodology that produced them. Standardized protocols in data collection, analysis, and interpretation are important properties of empirical research, and a healthy sign of a discipline's maturity. Further, standardization - intersubjective agreement between researchers on scientific procedures - and manualization - documenting and prescribing How in instruction manuals and handbooks to reduce the influence of Who, Where, and When on data quality - are fundamental to the objectivity of research as they guide scientists and facilitate the independence of a psychological experiment or test from basic boundary conditions. ${ }^{2}$ The extent to which researchers in a given field have been able to agree (either implicitly or explicitly) on these prescriptions is key to extracting meaningful inferences from meta-analytic effect size estimates.

\section{Terminology}

The steps to evaluate standardization of methodology to facilitate research synthesis proposed here are not inventing a wholly new meta-analytic procedure. Rather, they should be considered a specific type of literature review procedures that is typically overlooked in psychological meta-analyses, which are largely concerned with estimating the true distribution of effect sizes observed in primary research, and less with systematic searching, coding, and evaluating study methodology. Guidelines for systematic reviews in medicine (Higgins \& Green, 2011) and social sciences (The Campbell Collaboration, 2019) require giving substantial attention to critically evaluating the methodologies used in a literature and the limits these place on conclusions, recognizing that searching and evaluating studies is separate from meta-analytic modeling of study outcomes. Reporting guidelines for systematic reviews, e.g. PRISMA (Moher, Liberati, Tetzlaff, \& Altman, 2009) and specific to psychology MARS (Appelbaum et al., 2018), too, demand that meta-analysts describe how bias in both design and outcomes of individual studies was assessed, and how this assessment informed the synthesis itself. As such, this paper should be understood as an attempt at reintroducing these critical steps in the psychological research synthesis process.

Does that mean the suggestions made here are simply rephrasing the purpose and procedures of the "systematic review" part of meta-analyses? Although there certainly might be some redundancy when these steps are incorporated into the meta-analysis routine, particularly with regards to the systematic search and screening of primary studies, it may be valuable to coin a term referring specifically to the critical evaluation of the design and

\footnotetext{
${ }^{2}$ Notably, increasing standardization of research methods does not call for uniformity of research. Psychologists might (and should) use a host of different empirical approaches to the same research question. Many roads may lead to Rome, but there needs to be a mutual understanding of which one we call Via Appia.
} 
analytic choices made in a literature. Further, echoing Puljak (in press), methodological studies evaluating evidence are not necessarily systematic reviews. Whereas the purpose of the latter is to identify and pool all available empirical evidence (whose relevance is defined through predefined criteria) to answer a substantive research question, the aim of the former is to explore research practices and answering methodological questions without necessarily collating all empirical studies.

\section{Aims and Purpose}

Examining the degree of standardization is valuable to contextualize and evaluate observed meta-analytic effect sizes. In fact, standardization is, to some degree, a prerequisite of any meaningful research synthesis of a research literature. It informs researchers about the psychometric objectivity of results, the agreed definitions of constructs and their measurement, (the lack of) prevailing norms of what should be considered robust evidence, and the latent meaning of previous observations. Whereas meta-analyses in psychology are typically concerned with the synthesis of outcomes of studies (e.g., effect sizes), meta-method analyses dissect procedural decisions that deterministically lead to them (see Figure 1).

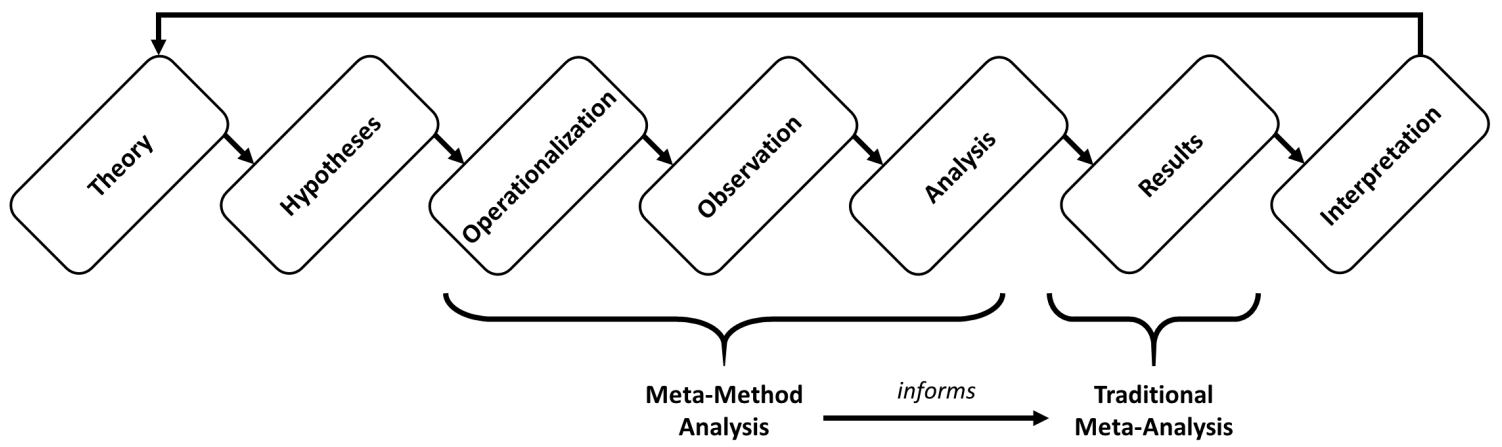

Figure 1. The empirical cycle. Traditionally, psychological meta-analyses systematically select study results to synthesize effect sizes from. Meta-method analysis informs this synthesis with an examination of the study corpus' operationalization, observation/measurement, and analysis methodologies.

A side-by-side comparison of procedural characteristics or analytical strategies of studies on the same research question, ideally using the same empirical paradigm, may reveal (1) idiosyncratic flexibility and peculiar deviations of individual research reports in areas with consistent disciplinary norms (for an example, see Simonsohn, 2013), or (2) endemic flexibility in areas where there is no standardization at all.

\section{Units of Analysis}

Any procedural study property and routine, such as operationalization, data collection, computation, analysis, or interpretation may be the subject of a systematic meta- 
method analysis. The extent to which performing a comprehensive meta-method analysis indicates the robustness and replicability of the body of research and informs the results of a meta-analysis naturally depends on the research question the latter is designed to answer. Whereas, for example, diverse samples and sampling methods might be considered a healthy sign of the literature on predictors of psychological well-being in the general population, substantial constraints would be expected in sampling methods of studies on the effectiveness of psychotherapy. Here the consistent application of narrowly defined inclusion criteria of study participants could even increase trust in the robustness of the literature.

Consequently, and with some irony, there may not be a standardized way of conducting a meta-method analysis, as (1) procedures and paradigms differ substantially between research domains, and (2) units of analysis may be relevant to some meta-analytic research question, but not others. Table 1 shows potential units of analysis with example references of publications (where available) reporting methodological examinations in that may inform meta-analyses in those respective areas, followed by two detailed examples further illustrating the usefulness of such efforts.

\section{Example 1: Aggressive Behavior}

The causes of aggressive behavior hypothesized by psychologists range from drugs, alcohol, and hot temperatures, to ostracism, frustration, and violent media (Sturmey, 2017). One of the most commonly used laboratory measures of aggressive behavior is the Competitive Reaction Time Task (CRTT), sometimes also called the Taylor Aggression Paradigm (TAP). In the CRTT, participants play a computerized reaction time game against another participant (actually a preprogrammed algorithm). At the beginning of each round, both players set the intensity of a noise blast, after which they are prompted to react to a stimulus as quickly as possible by pressing a button. The slower player is then punished with a noise blast using the intensity settings previously made by other player at the beginning of the round. The intensity settings are used as the measure for aggressive behavior.

While the CRTT ostensibly measures how much unpleasant, or even harmful, noise a participant is willing to administer to a (nonexistent) opponent, an examination of published studies in which the CRTT was used reveals a manifold of differences in the computational strategy or quantification by which an aggression score is extracted from the noise. Depending on how strictly one defines these differences, over 150 quantification strategies ${ }^{3}$ of aggression could be identified in 130 studies $^{4}$ (Elson, 2016; Elson, Mohseni, Breuer, Scharkow, \& Quandt, 2014).

\footnotetext{
${ }^{3}$ These procedural differences include the range of decibel levels, the availability of a nonaggressive response option, the sequence of wins and losses, the opponent's behavior (e.g., ambiguously or increasingly aggressive), the length of the test, the total number of trials, and which of those trials are included/excluded. Furthermore, there are studies in which a hypothetical CRTT was used (i.e. participants are given a description of the procedure and are then asked what volume/duration settings they would chose). Other times, electric shocks instead of noise blasts are used. All original research included here reported the CRTT as at least one round of a reaction time game in which the loser is punished with a noise blast set by the opponent. It is also possible that additional variations might be discovered in an examination of non-English publications and grey literature.

${ }^{4}$ As of March 31, 2018
} 
Table 1

A non-exhaustive list of potential units of analysis for meta-method analyses (with examples, if available).

\begin{tabular}{|c|c|c|}
\hline Procedure & Unit of Analysis & Example References \\
\hline Stimuli & $\begin{array}{l}\text { Presentation } \\
\text { Sequence }\end{array}$ & Wessel (2018); see Example 2 \\
\hline Sampling & $\begin{array}{l}\text { Statistical power } \\
\text { Recruitment } \\
\text { Sample characteristics } \\
\text { Population characteristics }\end{array}$ & Schimmack (2017) \\
\hline Operationalization & $\begin{array}{l}\text { Number of items/trials } \\
\text { Item formulation } \\
\text { Computational strategies }\end{array}$ & $\begin{array}{l}\text { Fiedler, Messner, and Bluemke } \\
(2006) \\
\text { Weidman, Steckler, and Tracy } \\
(2017) \\
\text { Credé, Harms, Niehorster, and } \\
\text { Gaye-Valentine (2012) } \\
\text { Weinstein (2018) } \\
\text { Elson, Mohseni, Breuer, Scharkow, } \\
\text { and Quandt (2014); see Example } 1\end{array}$ \\
\hline $\begin{array}{l}\text { Selection and filtering } \\
\text { of data points }\end{array}$ & $\begin{array}{l}\text { ROIs } \\
\text { Cut-offs and outliers }\end{array}$ & \\
\hline Analysis & $\begin{array}{l}\text { Analytical procedures } \\
\text { Critical parameters } \\
\text { Moderator variables }\end{array}$ & $\begin{array}{l}\text { Carp (2012a) } \\
\text { Harris, Chabot, and Mickes (2013) }\end{array}$ \\
\hline Interpretation & $\begin{array}{l}\text { Thresholds } \\
\text { Categorization }\end{array}$ & $\begin{array}{l}\text { Vul, Harris, Winkielman, and } \\
\text { Pashler (2009) }\end{array}$ \\
\hline
\end{tabular}

Note. In addition to systematically assessing the use of the above variables in psychological research, it can also be illuminating to consider the standards in reporting (see, for example, Carp, 2012b).

Not all of these procedures and quantification strategies differ substantially from each other (e.g., average volume of 25 trials vs average volume of 30 trials), and thus, choosing one over the other would not be expected to change a study's results dramatically. In other cases, however, differences are more apparent (e.g., logarithmized product of volume and duration of the first trial or highest volume across 30 trials), and their specific usage or theoretical fit (over others) is only occasionally justified in the respective publications. 


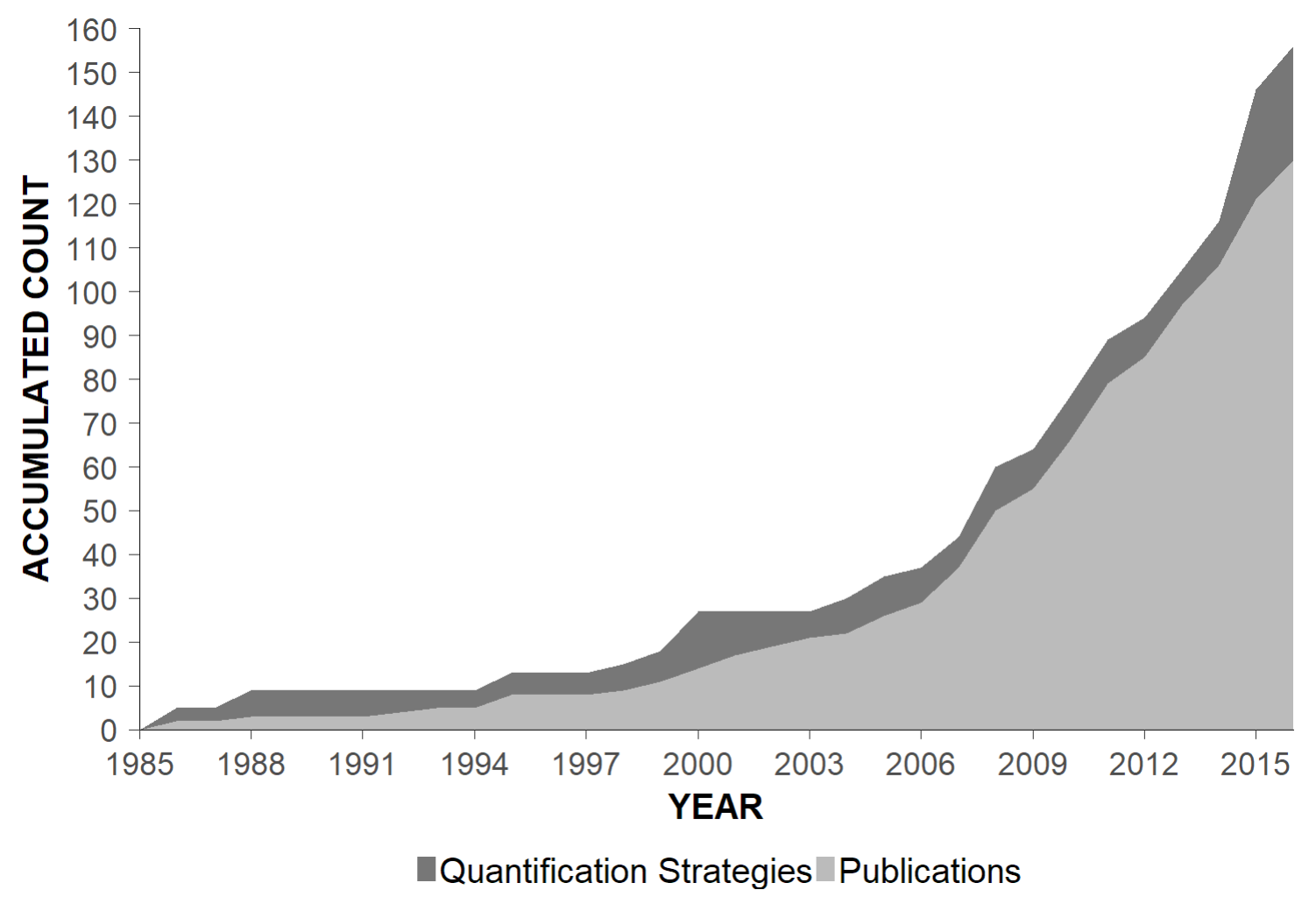

Figure 2. Accumulated growth of published studies with the CRTT and aggression score quantification strategies as of March 31, 2018.

Most quantification strategies (over 100) are just used once in a single research report and never reoccur in the literature; only three strategies have been used in more than 10 publications so far. Similarly, many studies reportedly rely on one or two quantification strategies, although there are publications that include up to 10. One potential explanation of these differences across studies it that due to a lack of manualization, researchers run the test with their own idiosyncratic settings. However, variability is also observed within-and not just between-individuals or labs using the CRTT in their research. In fact, it appears that a relatively small number of researchers have generated the majority of quantification strategies over time. Worryingly, the number of CRTT studies and the number of different quantification strategies appear to be accumulating almost linearly with time (see Figure 2).

Although arguably relevant, these considerations have hardly informed meta-analyses that include laboratory aggression studies. For example, in their meta-analysis on the link between playing violent video games and aggression, Anderson et al. (2010) highlighted the CRTT's prominence and validity as a behavioral measure. However, examining the included studies reveals that only a few, if any, of those studies that included the CRTT were identical with regard to their reported computational operationalization(s) of aggression (see Table $2)$. 
META-METHOD ANALYSIS

Table 2

English-language CRTT studies included in Anderson et al. (2010). ${ }^{a}$

\begin{tabular}{|c|c|c|c|c|}
\hline \multirow{2}{*}{$\begin{array}{l}\text { Study } \\
\text { Anderson et al. (2004) \#2 }\end{array}$} & \multicolumn{2}{|c|}{ Vol Dur 1} & & \multirow{2}{*}{$\begin{array}{l}\text { Reported Quantifications } \\
\text { Vol in trial } 1 \\
\text { Mean Vol in trial blocks } 2-9,10-17 \\
18-25\end{array}$} \\
\hline & $\checkmark$ & & 4 & \\
\hline Anderson et al. (2004) \#3 & $\checkmark$ & & 1 & Mean Vol \\
\hline Anderson et al. (2007) \#1 & $\checkmark$ & & 1 & $\begin{array}{l}\sqrt{N u m b e r} \text { of high Vol settings }(8-10) \\
\text { in } 25 \text { trials }\end{array}$ \\
\hline Anderson \& Carnagey (2009) \#3 & $\checkmark$ & & 2 & $\begin{array}{l}\text { Mean Vol } \\
\text { Number of high Vol settings }(8-10)\end{array}$ \\
\hline Anderson \& Dill (2000) \#2 & $\checkmark$ & $\checkmark$ & 4 & $\begin{array}{l}\text { Mean Dur (log-transformed) and } \\
\text { Mean Vol in } 12 \text { trials after winning } \\
\text { and losing each }\end{array}$ \\
\hline Anderson \& Murphy (2003) & $\checkmark$ & & 1 & Number of high Vol settings (8-10) \\
\hline Arriaga et al. (2008) & $\checkmark$ & $\checkmark$ & 1 & Mean $($ Vol + Dur $) / 2$ \\
\hline Bartholow et al. (2005) \#2 & $\checkmark$ & $\checkmark$ & 2 & $\begin{array}{l}\text { Mean Vol x Mean Dur } \\
\text { Vol x Dur in trial } 1\end{array}$ \\
\hline Bartholow \& Anderson (2002) \#1 & $\checkmark$ & & 8 & $\begin{array}{l}\text { Mean Vol } \\
\text { Mean Vol in trial blocks } 1-5,6-10, \\
11-15,16-20,21-25 \\
\text { Number of high Vol settings }(8-10) \\
\text { Log-transformed number of high Vol } \\
\text { settings (8-10) }\end{array}$ \\
\hline Carnagey \& Anderson (2005) \#3 & $\checkmark$ & & 1 & Mean Vol x $\sqrt{\text { Dur }}$ \\
\hline Ferguson et al. (2008) \#1 & $\checkmark$ & $\checkmark$ & 2 & Mean Vol and Mean Dur \\
\hline Konijn et al. (2007) & $\checkmark$ & & 2 & $\begin{array}{l}\text { Mean Vol } \\
\text { Vol in trial } 1\end{array}$ \\
\hline
\end{tabular}

Note. Study $=$ Reference with study number as reported in publication; $V o l=$ Noise volume reportedly set by subjects; Dur $=$ Noise duration reportedly set by subjects; $N=$ Number of quantifications used in analyses throughout the publication; Reported Quantifications = Quantification strategies used in analyses throughout the publication

a The quantification strategies reported in further Japanese-language publications included in Anderson et al. (2010) could not be identified due to the author's lack of education.

Implications for Meta-Analyses. Given that little is known about the validity of any reported quantification strategy, it is debatable whether a meaningful meta-analytic synthesis of this literature can actually be achieved in a meta-analysis of CRTT studies. There might simply be too much uncertainty regarding (1) the convergence of computational strategies on the underlying construct, and (2) the amount of bias introduced by this apparent flexibility. This, of course, has substantial implications for the informational value of various meta-analyses in the domain of aggression research where versions of the CRTT are routinely used (e.g., Anderson et al., 2010; Denson, O’Dean, Blake, \& Beames, 2018). 
Arguably, the magnitude of the problem with the use of multiple CRTT quantification strategies corresponds to their lack of convergence. Switching between or omitting quantification strategies would not lead to different outcomes in statistical analyses (and different inferences) if they were all perfectly correlated. However, if that were the case, it would be even more puzzling why so many different computational strategies are documented in the literature at all. Although typically, publication bias occurs through selective reporting of studies with statistically significant results, it is also possible that it results from selective reporting (and omission) of quantification strategies, particularly if multiple quantification strategies applied to the same data do not produce converging results. A recent study by Hyatt, Chester, Zeichner, and Miller (in press) examining bivariate correlations of various personality traits with 10 CRTT quantifications of the same data indicated directionally consistent relations with with moderate heterogeneity in effect sizes. Further validation studies will be required to enable aggression researchers making sense of the available literature.

One conclusion from this examination is that sharing of trial level CRTT data may ensure that studies are useful when a research community finally agrees on some standardized approach. Researchers are recommended to archive data from studies employing the CRTT on public repositories such as the Open Science Framework (www.osf.io) to allow independent testing of the assumptions required for meta-analytic synthesis. Furthermore, the extracted computational operationalizations may inspire "multiverse analyses" of CRTT studies, as suggested by Steegen, Tuerlinckx, Gelman, and Vanpaemel (2016). ${ }^{5}$

\section{Example 2: Inhibitory Control}

Whereas the previous example of a meta-method analysis revealed a lack of standardization in analytical strategies, this second one is concerned with procedural differences in data collection posing a potential concern for measurement validity.

Inhibitory control enables humans to rapidly cancel motor activity even after its initiation. It is a cognitive process and an important human capacity that permits an individual to inhibit their impulses or dominant behavioral responses to stimuli in order to select another (ideally more appropriate) response. Inhibitory control is involved in everyday decision making, such as stopping to cross the street when a car approaches or suppressing the urge to eat a diet-incongruent cake.

One of the most commonly used laboratory paradigms to study inhibitory control is the Go/No-Go Task. This task consists of a number of trials indicating either "Go" (respond, e.g., by pressing a button) or "No-Go" (do nothing). On each trial, prepotent motor activity ("Go") should first be elicited, which then sometimes has to be inhibited ("No-Go"). The response accuracy of each No-Go trial is used as a measure for inhibitory control. The most critical component in designing an inhibitory control task is to ensure that motor activity is elicited on each trial, so that No-Go trials truly test inhibitory control. To

\footnotetext{
${ }^{5}$ A "multiverse analysis" involves performing any number of reasonable analyses across a dataset to corroborate the robustness of a finding, which illustrates the consequences of arbitrariness in data processing and computational operationalizations.
} 
achieve this, two parameters of the experimental design are important: First, the relative proportion of Go and No-Go trials should be set so that No-Go trials are less frequent, making it strategically beneficial to initiate a Go response on every trial. Second, trials should be presented at a rapid pace, so that responses need to be made quickly.

The meta-method analysis of these two requirements in 241 studies using the Go/NoGo Task identified by Wessel (2018) revealed that, despite these well-known considerations, proportions of No-Go trials ranged from 6 to 60\%, and maximum trial durations ranged from 800 to 17,500 ms (see Figure 3). About 40\% of published Go/No-Go experiments had equiprobable Go and No-Go trials. Additionally, while most studies did present stimuli at a relatively rapid pace, almost $20 \%$ of studies used maximum trial durations greater than 4,000 ms, which allows a deliberate response to the stimulus instead of a prepotent response or its immediate inhibition. Most studies did not meet Wessel's (2018) recommended and empirically validated settings of (1) a No-Go trial proportion of less than $20 \%$ and (2) varying trial durations with a maximum of less than 1,500 ms (Elson, 2017; Wessel, 2018).

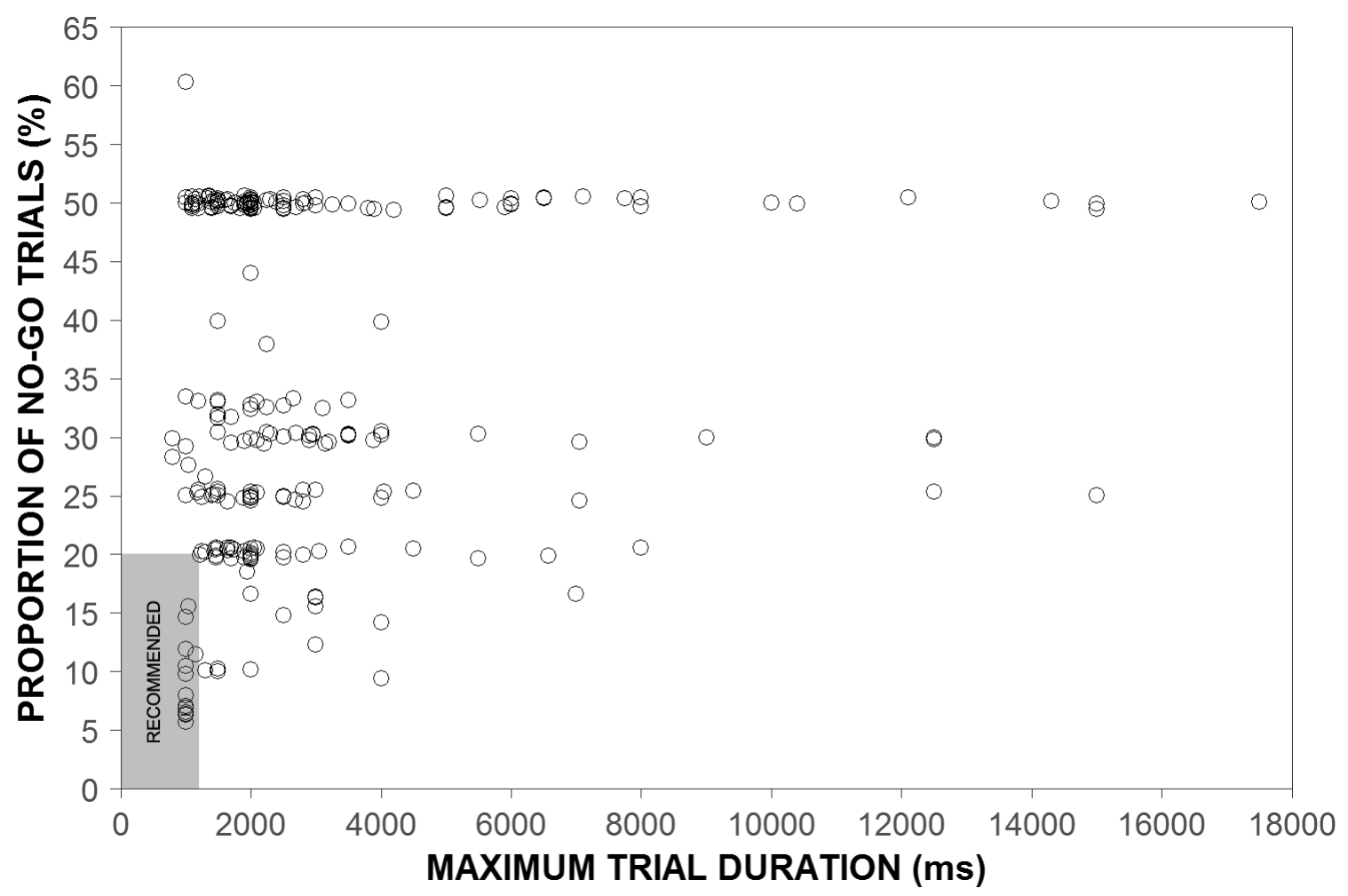

Figure 3. Proportions of No-Go trials and maximum trial durations in 241 studies using the Go/No-Go Task identified by Wessel (2018). Each circle represents one study. Slight jitter added for visibility.

Implication for Meta-Analyses. Studies with the Go/No-Go Task need to be carefully designed to ensure that inhibitory control is actually required to correctly perform during No-Go trials. If that is not the case, any differential effects that No-Go trials have in 
the context of such studies cannot be interpreted as effects of inhibitory control activity. It is unclear whether slow-paced and/or equiprobable Go/No-Go tasks elicit prepotent motor activity - and hence, whether they probe inhibitory control.

Consequently, meta-analyses including Go/No-Go studies would inherit this variability. Original studies with widely different settings may not actually assess a consistent, underlying effect or test the same theoretical prediction coherently, as only a proportion of the studies may have been designed appropriately to capture inhibitory control. The best scenario is that, without a meta-method analysis to select studies, the precision of meta-analytic effect size estimates is reduced to some extent; in the worst case, they are not meaningfully interpretable at all because the included studies actually operationalize different constructs.

\section{Planning a Meta-Method Analysis}

Psychologists seeking to complement their research with a meta-method analysis will have to make the (nontrivial) decision which of the potentially infinite number of unit(s) of analysis they should pick to substantively inform a meta-analysis. The principled, yet somewhat unsatisfying answer is that this should be consequentially determined by the meta-analytic research question. Several taxonomies could be employed to guide decision making, and one (useful) way emphasized here is to frame meta-method analyses on the continuum from confirmation to exploration:

The most obvious case may be a meta-analysis on a body of work in which prescription of empirical procedures should be inherent, either through highly manualized assessment batteries (e.g., intelligence tests) or, ideally, methodological specifications derived from formalized theories (which are, admittedly, currently quite rare in psychological science). Here, researchers may conduct a meta-method analysis to verify the (reported) conformity to those prescriptions, or whether deviations, if any, occur systematically.

In the absence of such clear norms, researchers may adopt a more pragmatic approach

and conduct a meta-method analysis with two questions in mind: (1) Which empirical procedures should be standardized across studies in order to allow a meaningful meta-analytic inference? A high degree of standardization (of those factors) would indicate intersubjective agreement on best practices, whereas a low degree might raise doubts about the replicability, generalizability, and synthesizability of observations. This type of meta-method analysis would provide researchers with the information whether a meta-analysis on a body of work could yield meaningful conclusions, and also lay the foundation for the establishment of gold standards. (2) Which empirical procedures might be prone to abuse of degrees of freedom to produce consistent results? Researchers may already suspect that flexibility in some (relevant) research practices may be more common than others (e.g., because they may be perceived as more acceptable by the community), and systematically search for confirmatory evidence. A high degree of consistency in those procedures would be a sign of transparency and reliability, whereas a low degree could indicate a body of literature is affected by questionable research practices. 
Finally, researchers may conduct a meta-method analysis on specific procedures out of genuine curiosity. The goal may not be to confirm studies are conforming to prescriptions, or to verify relevant study protocols, but to assess to which degree implicit agreement or field-specific traditions have already been established and shape study methodology.

\section{General Implications for Meta-Analyses}

Whether meta-method analysis is used as a quantitative or qualitative technique of research synthesis depends, again, on the meta-analytic research question it is applied to. However, there may be (at least) two important considerations to be taken into account when planning to incorporate its results into meta-analytic statistics (e.g., in form of a moderator analysis): (1) The greater the observed procedural differences across individual studies, the less studies per procedure are available, and consequently the lower the statistical power of such moderator tests. (2) As flexibility threatens the validity of studies, they may not consistently assess the same variable relationship, and as such, cannot be meaningfully included in the same analysis, even when the intent is to quantify the effect of methodological flexibility. ${ }^{6}$

Conversely, a meta-method analysis may reveal psychometric artefacts that can be controlled for statistically with appropriate meta-analytic correction techniques. Issues such as, for example, range restriction (Sackett \& Yang, 2000) or dichotomization of continuous variables (Hunter \& Schmidt, 1990) have predictable effects on observed studies, and the variability they introduce in meta-analytic effect size estimates can be adjusted for in a psychometric meta-analysis (Schmidt \& Hunter, 2015). Thus, depending on the specific findings of a meta-method analysis and the availability of appropriate adjustment techniques, it may be neither efficient nor appropriate to exclude studies from a meta-analysis, to use "methodological rigor" ratings of studies' as a moderator in a meta-analysis, or to conclude that a meta-analysis cannot be conducted at all due to inconsistent methodologies. Researchers are advised to distinguish methodological artefacts that are correctable (accounting for all assumptions and limitations of respective correction techniques themselves) and those that may be disqualifying factors limiting a research literature.

The most important implication of meta-method analyses is that they provide context required to meaningfully interpret observed meta-analytic effect size estimates. Metaanalytic studies in psychology are occasionally treated rather uncritically as the ultimate tool to produce conclusive final answers to a research question (Schmidt \& Oh, 2016). For example, Huesmann (2010) argues that Anderson et al.'s (2010) meta-analysis (see Example 1), although based partially on the documented, inconsistent use of a primary measure, is "[n]ailing the coffin shut on doubts that violent video games stimulate aggression" (p. 179). The value of a meta-method analysis could inform that interpretation of consistency of study outcomes in the context of unexplained flexibility in data collection - suggesting the coffin lid may actually still be somewhat ajar.

\footnotetext{
${ }^{6}$ An analysis of the predictors of automobile velocity with pilot weight as a moderator still requires all of the vehicles to be cars, and not occasionally a horse.
} 
Meta-method analyses could also improve the usefulness of meta-analyses in nascent fields where the number of studies is probably too low for a meaningful collation of the available evidence. Instead of attempting to (prematurely) provide conclusive answers to research questions, a systematic examination of methodological standards and norms would allow cautiously "taking stock" in the knowledge produced by a literature and its ability to provide meaningful conclusions. These types of analyses could be done on a small number of pioneer studies, steering fields in a productive direction before deficient empirical practices proliferate.

Beyond Standardization. It is noteworthy that, in this paper, meta-method analyses are somewhat narrowly framed as an approach to document and evaluate standardization, a critical property to maximize psychometric objectivity, across studies. However, it is entirely conceivable that a measure is used consistently in a literature without much available evidence for its construct or criterion validity (Weidman, Steckler, \& Tracy, 2017), or that common design choices (e.g., a relative overabundance of simple cross-sectional studies) limit the meta-analytic conclusions that can be drawn about causality and robustness of relationship between variables. Certainly, in such cases, a meta-method analysis concerned solely with issues of standardization would yield different, that is to say possibly less relevant conclusions than one with broader considerations.

There is no reason in principle why this rationale could not be applied to systematic, critical assessments of design and analytic choices made in a literature with regards to their meaning for psychometric reliability or validity (and, similarly, the validity of metaanalytic conclusions) instead of or in addition to mere consistency. The scope of this paper is not meant to discourage researchers from extending the general concept of meta-method analyses to higher-level psychometric properties. Rather, it emphasizes the importance of psychometric objectivity as a quality criterion and deterministic prerequisite to reliability and validity within classical test theory. Thus, this paper is meant to (re)introduce the basic concept of meta-method analyses to psychological research synthesis as the same time as reaffirming the foundational value of psychometric objectivity specifically, the and generally the importance of standardized protocols for meaningful cumulative research.

\section{General Implications for Primary Research}

Meta-method analyses, both in their conceptualization and their outcomes, have potential benefits for empirical studies: A more obvious one is that they may offer a systematic documentation of proven and tested empirical procedures and instructions for implementation in future study designs in that particular area (e.g., which stimulus material to use and to avoid, a decision tree for analytical strategies). This could, in turn, cumulatively increase the informational value of research synthesis.

As much as meta-method analyses benefit from clear, detailed descriptions of empirical studies, they may also shape transparent reporting standards as researchers seek to facilitate their effectiveness. Current reporting standards, such as the American Psychological Association's JARS (Appelbaum et al., 2018), already demand describing procedures in great detail. However, researchers (e.g. outside of the US) may not be aware of these 
guidelines, or if they are, they may find it difficult to apply broad recommendations to their specific research area as they ponder the granularity of their report. Surely, no researcher believes they are doing a bad job describing their study protocols, but they may disagree which information are relevant or should be made available, and where emphasis should be put. Although JARS specifically mentions the importance of transparent reporting so that scientific claims can be understood and evaluated, it may not be obvious which information are critical specifically to meaningful research synthesis.

Further, neither do journals routinely enforce compliance with these guidelines (other than style), nor is compliance systematically assessed. Thus, meta-method analyses may, at least in parts, document to which extent reporting practices in a given field already correspond to those standards, and point researchers to areas where they might be improved.

\section{Conclusion}

Meta-analyses are an accessible, systematic way of synthesizing and quantifying a body of research reports to answer overarching research questions. Their effectiveness is constrained by a range of challenges, such as biases (e.g., publication bias), methodology (e.g., appropriateness of search and selection procedures), and meta-methodology (e.g., disciplinary norms in empirical work). As meta-analyses necessarily inherit the quality of the included evidence, studies must meet a number of basic requirements in order to yield meaningful meta-analytic inferences. Aside from intersubjective agreement on terminology and fundamental theoretical constructs, empirical research benefits from rigorous best practices and gold standards. Fields in which researchers have yet been unable to agree on and develop useful procedural protocols might simply not be mature enough for a sophisticated synthesis (yet). Although tradition might be a weak form of protocol, it can still offer guidance. When there is no protocol for the procedures that many people are using, though, to what extent can individual studies be compared, combined, synthesized, and meta-analyzed? Researchers forming a community of practice need to figure out these details collectively before a meta-analysis (1) is potentially (mis)used to present research results as more conclusive than the underlying research standards could possibly allow, and (2) perpetuates empirical practice that may be degenerative to meaningful research synthesis and truth-seeking generally.

Conversely, the robustness and trustworthiness of meta-analyses in research domains in which emphasis is put on standardization and manualization would be further corroborated by meta-method analyses. Evidence of rigor across studies may (1) increase confidence in the replicability of individual studies and (2) precision of meta-analytic effect size estimates, (3) offer guidance to researchers entering a field as they look for tried-and-tested procedures, and (4) inspire systematic methodological research.

This article lays out an approach to research synthesis that can be used to contextualize meta-analytic effect size estimates conventionally reported in psychological research synthesis by systematically examining reported procedures that deterministically generate empirical outcomes. It is conservative in nature because it relies on what is being reported, and does not warrant inferring hidden causes (e.g., an inaccessible file drawer of studies) or 
biases occurring at a conceptual stage (Fiedler, 2011) which may not even be considered researcher degrees of freedom. This synergetic approach emphasizes the role of methods in advancing theory (Greenwald, 2012) by improving the quality of meta-analytic inferences. And as the quality of meta-analyses increase through these techniques, so will empirical standards in individual studies.

\section{References}

* Anderson, C. A. \& Carnagey, N. L. (2009). Causal effects of violent sports video games on aggression: Is it competitiveness or violent content? Journal of Experimental Social Psychology, 45(4), 731-739. doi:10.1016/j.jesp.2009.04.019

* Anderson, C. A., Carnagey, N. L., Flanagan, M., Arlin, B. J., Eubanks, J., \& Valentine, J. C. (2004). Violent video games: Specific effects of violent content on aggressive thoughts and behavior. In M. Zanna (Ed.), Advances in experimental social psychology (Vol. 36, pp. 199-249). New York, NY: Elsevier. doi:10.1016/S0065-2601(04)36004-1

* Anderson, C. A. \& Dill, K. E. (2000). Video games and aggressive thoughts, feelings, and behavior in the laboratory and in life. Journal of Personality and Social Psychology, 78(4), 772-790. doi:10.1037/0022-3514.78.4.772

* Anderson, C. A., Gentile, D. A., \& Buckley, K. E. (2007). Violent video game effects on children and adolescents: Theory, research, and public policy. New York, NY: Oxford University Press.

* Anderson, C. A. \& Murphy, C. R. (2003). Violent video games and aggressive behavior in young women. Aggressive Behavior, 29(5), 423-429. doi:10.1002/ab.10042

Anderson, C. A., Shibuya, A., Ihori, N., Swing, E. L., Bushman, B. J., Sakamoto, A., ... Saleem, M. (2010). Violent video game effects on aggression, empathy, and prosocial behavior in Eastern and Western countries: A meta-analytic review. Psychological Bulletin, 136(2), 151-173. doi:10.1037/a0018251

Appelbaum, M., Cooper, H., Kline, R. B., Mayo-Wilson, E., Nezu, A. M., \& Rao, S. M. (2018). Journal article reporting standards for quantitative research in psychology: The APA Publications and Communications Board task force report. American Psychologist, 73 (1), 3-25. doi:10.1037/amp0000191

* Arriaga, P., Esteves, F., Caneiro, P., \& Monteiro, M. B. (2008). Are the effects of Unreal violent video games pronounced when playing with a virtual reality system? Aggressive Behavior, 34(5), 521-538. doi:10.1002/ab.20272

* Bartholow, B. D. \& Anderson, C. A. (2002). Effects of violent video games on aggressive behavior: Potential sex differences. Journal of Experimental Social Psychology, 38(3), 283-290. doi:10.1006/jesp.2001.1502

* Bartholow, B. D., Sestir, M. A., \& Davis, E. B. (2005). Correlates and consequences of exposure to video game violence: Hostile personality, empathy, and aggressive behavior. Personality and Social Psychology Bulletin, 31(11), 1573-1586. doi:10.1177/ 0146167205277205

* Carnagey, N. L. \& Anderson, C. A. (2005). The effects of reward and punishment in violent video games on aggressive affect, cognition, and behavior. Psychological Science, 16(11), 882-889. doi:10.1111/j.1467-9280.2005.01632.x 
Carp, J. (2012a). On the plurality of (methodological) worlds: Estimating the analytic flexibility of fMRI experiments. Frontiers in Neuroscience, 6, 149. doi:10.3389/fnins. 2012.00149

Carp, J. (2012b). The secret lives of experiments: Methods reporting in the fMRI literature. NeuroImage, 63(1), 289-300. doi:10.1016/j.neuroimage.2012.07.004

Carter, E., Schönbrodt, F., Gervais, W., \& Hilgard, J. (in press). Correcting for bias in psychology: A comparison of meta-analytic methods. Advances in Methods and Practices in Psychological Science.

Credé, M., Harms, P., Niehorster, S., \& Gaye-Valentine, A. (2012). An evaluation of the consequences of using short measures of the Big Five personality traits. Journal of Personality and Social Psychology, 102(4), 874-888. doi:10.1037/a0027403

Denson, T. F., O’Dean, S. M., Blake, K. R., \& Beames, J. R. (2018). Aggression in women: Behavior, brain and hormones. Frontiers in Behavioral Neuroscience, 12, 81. doi:10. $3389 /$ fnbeh.2018.00081

Duval, S. \& Tweedie, R. (2000). Trim and Fill: A simple funnel-plot-based method of testing and adjusting for publication bias in meta-analysis. Biometrics, 56 (2), 455-463. doi:10. 1111/j.0006-341X.2000.00455.x

Ellis, P. D. (2010). The essential guide to effect sizes. Statistical power, meta-analysis, and the interpretation of research results. Cambridge, UK: Cambridge University Press.

Elson, M. (2016). FlexibleMeasures.com: Competitive Reaction Time Task. doi:10.17605/ OSF.IO/4G7FV

Elson, M. (2017). FlexibleMeasures.com: Go/No-Go Task. doi:10.17605/OSF.IO/GSX52

Elson, M., Mohseni, M. R., Breuer, J., Scharkow, M., \& Quandt, T. (2014). Press CRTT to measure aggressive behavior: The unstandardized use of the Competitive Reaction Time Task in aggression research. Psychological Assessment, 26(2), 419-432. doi:10. $1037 / \mathrm{a} 0035569$

* Ferguson, C. J. (2008). The school shooting/violent video game link: Causal relationship or moral panic? Journal of Investigative Psychology and Offender Profiling, 5(1-2), 25-37. doi:10.1002/jip.76

Ferguson, C. J. \& Heene, M. (2012). A vast graveyard of undead theories: Publication bias and psychological science's aversion to the null. Perspectives on Psychological Science, 7(6), 555-561. doi:10.1177/1745691612459059

Fiedler, K. (2011). Voodoo correlations are everywhere - Not only in neuroscience. Perspectives on Psychological Science, 6(2), 163-171. doi:10.1177/1745691611400237

Fiedler, K., Messner, C., \& Bluemke, M. (2006). Unresolved problems with the "I", the "A", and the "T": A logical and psychometric critique of the Implicit Association Test (IAT). European Review of Social Psychology, 17(1), 74-147. doi:10.1080/10463280600681248

Field, A. \& Gillett, R. (2010). How to do a meta-analysis. The British Journal of Mathematical and Statistical Psychology, 63(3), 665-694. doi:10.1348/000711010X502733

Gignac, G. E. \& Szodorai, E. T. (2016). Effect size guidelines for individual differences researchers. Personality and Individual Differences, 102, 74-78. doi:10.1016/j.paid. 2016.06.069

Greenwald, A. G. (2012). There is nothing so theoretical as a good method. Perspectives on Psychological Science, 7(2), 99-108. doi:10.1177/1745691611434210 
Hagger, M. S., Chatzisarantis, N. L. D., Alberts, H., Anggono, C. O., Batailler, C., Birt, A., ... Zwienenberg, M. (2016). A multilab preregistered replication of the egodepletion effect. Perspectives on Psychological Science, 11(4), 546-573. doi:10.1177/ 1745691616652873

Harris, C. R., Chabot, A., \& Mickes, L. (2013). Shifts in methodology and theory in menstrual cycle research on attraction. Sex Roles, 69(9-10), 525-535. doi:10.1007/s11199013-0302-3

Higgins, J. P. T. \& Green, S. (Eds.). (2011). Cochrane handbook for systematic reviews of interventions (version 5.1.0). The Cochrane Collaboration. Retrieved from www. handbook.cochrane.org

Huesmann, L. R. (2010). Nailing the coffin shut on doubts that violent video games stimulate aggression: Comment on Anderson et al. (2010). Psychological Bulletin, 136(2), 179181. doi: $10.1037 / \mathrm{a} 0018567$

Hunter, J. E. \& Schmidt, F. L. (1990). Dichotomization of continuous variables: The implications for meta-analysis. Journal of Applied Psychology, 75(3), 334-349. doi:10. 1037/0021-9010.75.3.334

Hyatt, C. S., Chester, D. S., Zeichner, A., \& Miller, J. D. (in press). Analytic flexibility in laboratory aggression paradigms: Relations with personality traits vary (slightly) by operationalization of aggression. Aggressive Behavior. doi:10.1002/ab.21830

John, L. K., Loewenstein, G., \& Prelec, D. (2012). Measuring the prevalence of questionable research practices with incentives for truth telling. Psychological Science, 23(5), 524532. doi:10.1177/0956797611430953

* Konijn, E. A., Nije Bijvank, M., \& Bushman, B. J. (2007). I wish I were a warrior: The role of wishful identification in the effects of violent video games on aggression in adolescent boys. Developmental Psychology, 43(4), 1038-1044. doi:10.1037/0012-1649.43.4.1038

Kühberger, A., Fritz, A., \& Scherndl, T. (2014). Publication bias in psychology: A diagnosis based on the correlation between effect size and sample size. PLOS ONE, 9(9), e105825. doi:10.1371/journal.pone.0105825

Lakens, D. \& Evers, E. R. K. (2014). Sailing from the seas of chaos into the corridor of stability: Practical recommendations to increase the informational value of studies. Perspectives on Psychological Science, 9(3), 278-292. doi:10.1177/1745691614528520

LeBel, E. P., Borsboom, D., Giner-Sorolla, R., Hasselman, F., Peters, K. R., Ratliff, K. A., \& Smith, C. T. (2013). PsychDisclosure.org: Grassroots support for reforming reporting standards in psychology. Perspectives on Psychological Science, 8(4), 424-432. doi:10. $1177 / 1745691613491437$

LeBel, E. P., McCarthy, R. J., Earp, B. D., Elson, M., \& Vanpaemel, W. (2018). A unified framework to quantify the credibility of scientific findings. Advances in Methods and Practices in Psychological Science, 1(3), 389-402. doi:10.1177/2515245918787489

McShane, B. B., Böckenholt, U., \& Hansen, K. T. (2016). Adjusting for publication bias in meta-analysis. Perspectives on Psychological Science, 11(5), 730-749. doi:10.1177/ 1745691616662243

Moher, D., Liberati, A., Tetzlaff, J., \& Altman, D. G. (2009). Preferred reporting items for systematic reviews and meta-analyses: The PRISMA statement. BMJ, 339, b2535. doi:10.1136/bmj.b2535 
Nelson, L. D., Simmons, J., \& Simonsohn, U. (2018). Psychology's renaissance. Annual Review of Psycholgy, 69, 511-534. doi:10.1146/annurev-psych-122216-011836

Nosek, B. A. \& Bar-Anan, Y. (2012). Scientific utopia: I. Opening scientific communication. Psychological Inquiry, 23(3), 217-243. doi:10.1080/1047840X.2012.692215

Nosek, B. A., Spies, J. R., \& Motyl, M. (2012). Scientific utopia: II. Restructuring incentives and practices to promote truth over publishability. Perspectives on Psychological Science, 7(6), 615-631. doi:10.1177/1745691612459058

Nuijten, M. B., Hartgerink, C. H. J., van Assen, M. A. L. M., Epskamp, S., \& Wicherts, J. M. (2016). The prevalence of statistical reporting errors in psychology (1985-2013). Behavior Research Methods, 48(4), 1205-1226. doi:10.3758/s13428-015-0664-2

Open Science Collaboration. (2015). Estimating the reproducibility of psychological science. Science, 349 (6251), aac4716. doi:10.1126/science.aac4716

Plonsky, L. \& Oswald, F. L. (2014). How big is "big"? Interpreting effect sizes in L2 research. Language Learning, 64(4), 878-912. doi:10.1111/lang.12079

Puljak, L. (in press). Methodological studies evaluating evidence are not systematic reviews. Journal of Clinical Epidemiology. doi:10.1016/j.jclinepi.2019.02.002

Renkewitz, F. \& Keiner, M. (2018). How to detect publication bias in psychological research? A comparative evaluation of six statistical methods. PsyArXiv. doi:10.31234/osf.io/ w94ep

Sackett, P. R. \& Yang, H. (2000). Correction for range restriction: An expanded typology. Journal of Applied Psychology, 85(1), 112-118. doi:10.1037/0021-9010.85.1.112

Schimmack, U. (2014). The test of insufficient variance (TIVA): A new tool for the detection of questionable research practices. Retrieved from http://replicationindex.wordpress. com

Schimmack, U. (2017). The power of the pen paradigm: A replicability analysis. Retrieved from http://replicationindex.wordpress.com

Schmidt, F. L. \& Hunter, J. E. (2015). Methods of meta-analysis: Correcting error and bias in research findings (3rd). London: Sage. doi:10.4135/9781483398105

Schmidt, F. L. \& Oh, I.-S. (2016). The crisis of confidence in research findings in psychology: Is lack of replication the real problem? Or is it something else? Archives of Scientific Psychology, 4 (1), 32-37. doi:10.1037/arc0000029

Schönbrodt, F. D. (2016). p-hacker: Train your p-hacking skills! Retrieved from http:// shinyapps.org/apps/p-hacker/

Simonsohn, U. (2013). Just post it: The lesson from two cases of fabricated data detected by statistics alone. Psychological Science, 24(10), 1875-1888. doi:10.1177/ 0956797613480366

Simonsohn, U., Nelson, L. D., \& Simmons, J. P. (2014a). p-curve: A key to the file-drawer. Journal of Experimental Psychology: General, 143(2), 534-547. doi:10.1037/a0033242

Simonsohn, U., Nelson, L. D., \& Simmons, J. P. (2014b). p-curve and effect size: Correcting for publication bias using only significant results. Perspectives on Psychological Science, 9(6), 666-681. doi:10.1177/1745691614553988

Stanley, T. D. \& Doucouliagos, H. (2014). Meta-regression approximations to reduce publication selection bias. Research Synthesis Methods, 5(1), 60-78. doi:10.1002/jrsm.1095 
Steegen, S., Tuerlinckx, F., Gelman, A., \& Vanpaemel, W. (2016). Increasing transparency through a multiverse analysis. Perspectives on Psychological Science, 11(5), 702-712. doi:10.1177/1745691616658637

Sturmey, P. (Ed.). (2017). The Wiley handbook of violence and aggression. Hoboken, NJ: Wiley.

Sumner, P., Vivian-Griffiths, S., Boivin, J., Williams, A., Venetis, C. A., Davies, A., ... Chambers, C. D. (2014). The association between exaggeration in health related science news and academic press releases: Retrospective observational study. BMJ, 349, g7015. doi:10.1136/bmj.g7015

Szucs, D. \& Ioannidis, J. P. A. (2017). Empirical assessment of published effect sizes and power in the recent cognitive neuroscience and psychology literature. PLOS Biology, 15(3), e2000797. doi:10.1371/journal.pbio.2000797

The Campbell Collaboration. (2019). Campbell systematic reviews: Policies and guidelines. Oslo, Norway. doi:10.4073/cpg.2016.1

van Assen, M. A. L. M., van Aert, R. C. M., \& Wicherts, J. M. (2014). Meta-analysis using effect size distributions of only statistically significant studies. Psychological Methods. doi: $10.1037 /$ met0000025

Vul, E., Harris, C., Winkielman, P., \& Pashler, H. (2009). Puzzlingly high correlations in fMRI studies of emotion, personality, and social cognition. Perspectives on Psychological Science, 4(3), 274-290. doi:10.1111/j.1745-6924.2009.01125.x

Wagenmakers, E.-J., Beek, T., Dijkhoff, L., Gronau, Q. F., Acosta, A., Adams, R. B., ... Zwaan, R. A. (2016). Registered replication report: Strack, Martin, \& Stepper (1988). Perspectives on Psychological Science, 11(6), 917-928. doi:10.1177/1745691616674458

Wagenmakers, E.-J., Wetzels, R., Borsboom, D., \& van der Maas, H. L. J. (2011). Why psychologists must change the way they analyze their data: The case of Psi: Comment on Bem (2011). Journal of Personality and Social Psychology, 100 (3), 426-432. doi:10. $1037 / \mathrm{a} 0022790$

Wagenmakers, E.-J., Wetzels, R., Borsboom, D., van der Maas, H. L. J., \& Kievit, R. A. (2012). An agenda for purely confirmatory research. Perspectives on Psychological Science, 7(6), 632-638. doi:10.1177/1745691612463078

Weidman, A. C., Steckler, C. M., \& Tracy, J. L. (2017). The jingle and jangle of emotion assessment: Imprecise measurement, casual scale usage, and conceptual fuzziness in emotion research. Emotion, 17(2), 267-295. doi:10.1037/emo0000226

Weinstein, Y. (2018). Mind-wandering, how do I measure thee with probes? Let me count the ways. Behavior Research Methods, 50(2), 642-661. doi:10.3758/s13428-017-0891-9

Wessel, J. R. (2018). Prepotent motor activity and inhibitory control demands in different variants of the go/no-go paradigm. Psychophysiology, 55(3), e12871. doi:10.1111/ psyp. 12871

Wicherts, J. M., Bakker, M., \& Molenaar, D. (2011). Willingness to share research data is related to the strength of the evidence and the quality of reporting of statistical results. PLOS ONE, 6(11), e26828. doi:10.1371/journal.pone.0026828

Wicherts, J. M., Kievit, R. A., Bakker, M., \& Borsboom, D. (2012). Letting the daylight in: Reviewing the reviewers and other ways to maximize transparency in science. Frontiers in Computational Neuroscience, 6, No. 20. doi:10.3389/fncom.2012.00020 
Wiernik, B. M., Kostal, J. W., Wilmot, M. P., Dilchert, S., \& Ones, D. S. (2017). Empirical benchmarks for interpreting effect size variability in meta-analysis. Industrial and Organizational Psychology, 10(3), 472-479. doi:10.1017/iop.2017.44

Note: All references from Example 2 are marked with an * 